\title{
An Average Linear Difference Scheme for the Generalized Rosenau-KdV Equation
}

\author{
Maobo Zheng ${ }^{1}$ and Jun Zhou ${ }^{2}$ \\ ${ }^{1}$ Chengdu Technological University, Chengdu 610031, China \\ ${ }^{2}$ School of Mathematics and Computer Science, Yangtze Normal University, Chongqing 408100, China \\ Correspondence should be addressed to Jun Zhou; lmzjzgoi@sohu.com
}

Received 14 June 2013; Accepted 7 January 2014; Published 25 February 2014

Academic Editor: Anjan Biswas

Copyright (c) 2014 M. Zheng and J. Zhou. This is an open access article distributed under the Creative Commons Attribution License, which permits unrestricted use, distribution, and reproduction in any medium, provided the original work is properly cited.

An average linear finite difference scheme for the numerical solution of the initial-boundary value problem of Generalized Rosenau$\mathrm{KdV}$ equation is proposed. The existence, uniqueness, and conservation for energy of the difference solution are proved by the discrete energy norm method. It is shown that the finite difference scheme is 2nd-order convergent and unconditionally stable. Numerical experiments verify that the theoretical results are right and the numerical method is efficient and reliable.

\section{Introduction}

$\mathrm{KdV}$ equation has been used in very wide applications and undergone research which can be used to describe wave propagation and spread interaction as follows [1-4]:

$$
u_{t}+u u_{x}+u_{x x x}=0 .
$$

In the study of the dynamics of dense discrete systems, the case of wave-wave and wave-wall interactions cannot be described using the well-known $\mathrm{KdV}$ equation. To overcome this shortcoming of the $\mathrm{KdV}$ equation, Rosenau $[5,6]$ proposed the so-called Rosenau equation:

$$
u_{t}+u_{x}+u u_{x}+u_{x x x x t}=0 .
$$

The existence and the uniqueness of the solution for (2) were proved by Park [7]. But it is difficult to find the analytical solution for (2). Since then, much work has been done on the numerical method for (2) ([8-13] and also the references therein). On the other hand, for the further consideration of the nonlinear wave, the viscous term $u_{x x x}$ needs to be included [14]:

$$
u_{t}+u_{x}+u_{x x x}+u_{x x x x t}+u u_{x}=0 .
$$

This equation is usually called the Rosenau-KdV equation. Zuo [14] discussed the solitary wave solutions and periodic solutions for Rosenau-KdV equation. In [15], a conservative linear finite difference scheme for the numerical solution for an initial-boundary value problem of Rosenau$\mathrm{KdV}$ equation is considered. In this paper, we consider the following Generalized Rosenau-KdV equation:

$$
u_{t}+u_{x}+u_{x x x}+u_{x x x x t}+\left(u^{p}\right)_{x}=0,
$$

where $p \geq 2$ is an integer. When $p=2$, (4) is called usual Rosenau-KdV (3).

In $[16,17]$, authors discussed the solitary solutions for the Generalized Rosenau-KdV equation with usual solitary ansatz method. The authors also gave the two invariants for the Generalized Rosenau-KdV equation. In particular, in [17], the authors not only studied the two types of soliton solution, one is solitary wave solution and the other is singular soliton. Furthermore, they also used the perturbation theory and the semivariation principle to study the perturbed Generalized Rosenau-KdV equation analytically. In [18], only ansatz method was applied to obtain the topological soliton solution or the shock solution of this equation. Three methods, ansatz method, $G^{\prime} / G$-expansion method, and the exp-function method, were applied to extract a few more solutions to this equation in [19].

As we all know, most of the time, we need to think of the numerical solution of nonlinear evolution equations. Many 
scholars in this field have a good work. In [20], the authors simulate the numerical solution of the Klein-Gordon equation by using the spectral method where rational Chebyshev functions are used as basic functions. In [21], the authors study the numerical solution of the two-dimensional SineGordon equation (SGE) using a split-step Chebyshev Spectral Method. In [22], the authors develop a Galerkin spectral technique for computing localized solutions of equation with Sixth-Order Generalized Boussinesq Equation (6GBE). In [15], the authors propose a conservative three-level linear finite difference scheme with second-order convergent for the numerical solution of the initial-boundary value problem of Rosenau-KdV equation.

But the numerical method of the initial-boundary value problem of Generalized Rosenau-KdV equation has not been studied till now. In this paper, we propose an average threelevel linear finite difference scheme for (4) with the boundary conditions

$$
\begin{gathered}
u\left(X_{l}, t\right)=u\left(X_{r}, t\right)=0, \quad u_{x}\left(X_{l}, t\right)=u_{x}\left(X_{r}, t\right)=0, \\
u_{x x}\left(X_{l}, t\right)=u_{x x}\left(X_{r}, t\right)=0, \quad t \in[0, T]
\end{gathered}
$$

and initial condition

$$
u(x, 0)=u_{0}(x) .
$$

The initial-boundary value problem (3)-(5) possesses the following conservative properties $[16,17]$ :

$$
\begin{gathered}
M(t)=\int_{X_{l}}^{X_{r}} u d x=\int_{X_{l}}^{X_{r}} u_{0} d x=M(0), \\
E(t)=\int_{X_{l}}^{X_{r}}\left(u^{2}+u_{x x}^{2}\right) d x=\|u\|_{L_{2}}^{2}+\left\|u_{x x}\right\|_{L_{2}}^{2}=E(0) .
\end{gathered}
$$

When $X_{l} \ll 0, X_{r} \gg 0$, the initial-boundary value problem (4)-(6) and the Cauchy problem (4) are consistent, so the boundary conditions (5) are reasonable.

Compared to the implicit C-N nonlinear scheme, the scheme in this paper is linear and it can reduce computing cost. We will prove existence, uniqueness, and stability of the numerical solution. The studies show that the convergence of the scheme is 2 nd-order rate. The most important point is that the scheme is conservative for energy.

The rest of this paper is organized as follows. In Section 2, we propose a three-level average implicit linear finite difference scheme for Generalized Rosenau-KdV equation and discuss the discrete conservative properties for energy. In Section 3, we prove that the scheme is uniquely solvable. In Section 4 , we prove that the finite difference scheme is 2 ndorder convergent and unconditionally stable. In Section 5, we give some numerical simulation to verify our theoretical analysis. Finally, in Section 6, we get our conclusion.

\section{Difference Scheme and Some Properties of Its Solution}

In this section, we first give some notation which will be used in this paper and propose an average linear difference scheme for the problem of (4)-(6).
As usual, denote $x_{j}=X_{l}+j h, t_{n}=n \tau, 0 \leq j \leq J, 0 \leq n \leq$ $N$, where $h=\left(X_{r}-X_{l}\right) / J$ and let $\tau$ be the uniform, the spatial, and the temporal step size, respectively. Let $u_{j}^{n} \approx u(j h, n \tau)$, $Z_{h}^{0}=\left\{u=\left(u_{j}\right) \mid u_{-1}=u_{0}=u_{J}=u_{J+1}=0,-1 \leq j \leq J+1\right\}$. Throughout this paper, we will denote $C$ as a generic constant independent of $h$ and $\tau$ that varies in the context.

We define the difference operators, inner product, and norms that will be used in this paper as follows:

$$
\begin{gathered}
\left(u_{j}^{n}\right)_{x}=\frac{u_{j+1}^{n}-u_{j}^{n}}{h}, \quad\left(u_{j}^{n}\right)_{\bar{x}}=\frac{u_{j}^{n}-u_{j-1}^{n}}{h}, \\
\left(u_{j}^{n}\right)_{\widehat{x}}=\frac{u_{j+1}^{n}-u_{j-1}^{n}}{2 h}, \quad\left(u_{j}^{n}\right)_{t}=\frac{u_{j}^{n+1}-u_{j}^{n}}{\tau}, \\
\left(u_{j}^{n}\right)_{\hat{t}}=\frac{u_{j}^{n+1}-u_{j}^{n-1}}{2 \tau}, \quad\left(u_{j}^{n}\right)_{x \bar{x}}=\frac{u_{j+1}^{n}-2 u_{j}^{n}+u_{j-1}^{n}}{h^{2}}, \\
\bar{u}_{j}^{n}=\frac{u_{j}^{n+1}+u_{j}^{n-1}}{2}, \quad u_{j}^{n+(1 / 2)}=\frac{u_{j}^{n+1}+u_{j}^{n}}{2}, \\
\left\langle u^{n}, v^{n}\right\rangle=h \sum_{j=1}^{J-1} u_{j}^{n} v_{j}^{n}, \quad\left\|u^{n}\right\|^{2}=\left\langle u^{n}, u^{n}\right\rangle, \\
\left\|u^{n}\right\|_{\infty}=\max _{0 \leq j \leq J-1}\left|u_{j}^{n}\right| \cdot
\end{gathered}
$$

Since $\left(u^{p}\right)_{x}=(p /(p+1))\left[u^{p-1} u_{x}+\left(u^{p}\right)_{x}\right]$, the following finite difference scheme for the problem $(4)-(6)$ is considered:

$$
\begin{gathered}
\left(u_{j}^{n}\right)_{\hat{t}}+\left(\bar{u}_{j}^{n}\right)_{\widehat{x}}+\left(\bar{u}_{j}^{n}\right)_{x \bar{x} \hat{x}}+\left(u_{j}^{n}\right)_{x x \bar{x} \bar{x} \hat{t}} \\
+\frac{p}{p+1}\left\{\left(u_{j}^{n}\right)^{p-1}\left(\bar{u}_{j}^{n}\right)_{\widehat{x}}+\left[\left(u_{j}^{n}\right)^{p-1} \bar{u}_{j}^{n}\right]_{\widehat{x}}\right\}=0 \\
u_{j}^{0}=u_{0}\left(x_{j}\right), \quad 1 \leq j \leq J-1 \\
u_{0}^{n}=u_{J}^{n}=0, \quad\left(u_{0}^{n}\right)_{\widehat{x}}=\left(u_{J}^{n}\right)_{\widehat{x}}=0 \\
\left(u_{0}^{n}\right)_{x \bar{x}}=\left(u_{J}^{n}\right)_{\bar{x} x}=0 .
\end{gathered}
$$

Lemma 1 (see [23]). For any two mesh functions, $u, v \in Z_{h}^{0}$, one has

$$
\begin{gathered}
\left\langle v_{x}, u\right\rangle=-\left\langle v, u_{\bar{x}}\right\rangle, \quad\left\langle u_{\hat{x}}, v\right\rangle=-\left\langle u, v_{\hat{x}}\right\rangle, \\
\left\langle u, v_{x \bar{x}}\right\rangle=-\left\langle u_{x}, v_{x}\right\rangle .
\end{gathered}
$$

Then we have

$$
\left\langle u, u_{x \bar{x}}\right\rangle=-\left\langle u_{x}, u_{x}\right\rangle=-\left\|u_{x}\right\|^{2} .
$$

Furthermore, if $\left(u_{0}^{n}\right)_{x \bar{x}}=\left(u_{J}^{n}\right)_{x \bar{x}}=0$, then

$$
\left\langle u, u_{x x \bar{x} \bar{x}}\right\rangle=\left\|u_{x x}\right\|^{2} .
$$

Lemma 2. Suppose that $u \in H_{0}^{2}\left[X_{l}, X_{r}\right]$; then the solution of the initial-boundary value problem (4)-(6) satisfies

$$
\|u\|_{L_{2}} \leq C, \quad\left\|u_{x}\right\|_{L_{2}} \leq C, \quad\|u\|_{\infty} \leq C .
$$


Proof. It follows from the conservative law (8) that we get

$$
\|u\|_{L_{2}} \leq C, \quad\left\|u_{x x}\right\|_{L_{2}} \leq C
$$

Using part integration method, Hölder inequality, and Schwartz inequality, we get

$$
\begin{aligned}
\left\|u_{x}\right\|_{L_{2}}^{2} & =\int_{X_{l}}^{X_{r}} u_{x} u_{x} d x=\left.u u_{x}\right|_{X_{l}} ^{X_{r}}-\int_{X_{r}}^{X_{r}} u u_{x x} d x \\
& =-\int_{X_{l}}^{X_{r}} u u_{x x} d x \leq\|u\|_{L_{2}}\left\|u_{x x}\right\|_{L_{2}} \\
& \leq \frac{1}{2}\left(\|u\|_{L_{2}}^{2}+\left\|u_{x x}\right\|_{L_{2}}^{2}\right) .
\end{aligned}
$$

Hence, $\left\|u_{x}\right\|_{L_{2}} \leq C$. According to Sobolev's inequality, we have $\|u\|_{\infty} \leq C$.

Theorem 3. Supposing $u_{0} \in H_{0}^{2}\left[X_{l}, X_{r}\right]$, then the scheme (10)-(12) is conservative for discrete energy; that is,

$$
\begin{aligned}
E^{n} & =\frac{1}{2}\left(\left\|u^{n+1}\right\|^{2}+\left\|u^{n}\right\|^{2}\right)+\frac{1}{2}\left(\left\|u_{x x}^{n+1}\right\|^{2}+\left\|u_{x x}^{n}\right\|^{2}\right) \\
& =E^{n-1}=\cdots=E^{0} .
\end{aligned}
$$

Proof. Computing the inner product of (10) with $2 \bar{u}^{n}$ (i.e., $u^{n+1}+u^{n-1}$ ), we have

$$
\begin{aligned}
h \sum_{j=1}^{J-1}\{ & \frac{1}{2 \tau}\left(u_{j}^{n+1}-u_{j}^{n-1}\right) \cdot 2 \bar{u}_{j}^{n}+\left(\left(\bar{u}_{j}^{n}\right)_{\widehat{x}} \cdot 2 \bar{u}_{j}^{n}\right) \\
& +\left(\left(\bar{u}_{j}^{n}\right)_{x \bar{x} \hat{x}} \cdot 2 \bar{u}_{j}^{n}\right)+\frac{1}{2 \tau}\left(\left(u_{j}^{n+1}\right)_{x x \bar{x} \bar{x}}-\left(u_{j}^{n-1}\right)_{x x \bar{x} \bar{x}}\right) \cdot 2 \bar{u}_{j}^{n} \\
& \left.+\left(P_{j} \cdot 2 \bar{u}_{j}^{n}\right)\right\}=0,
\end{aligned}
$$

where

$$
P_{j}=\frac{p}{p+1}\left\{\left(u_{j}^{n}\right)^{p-1} \cdot\left(\bar{u}_{j}^{n}\right)_{\widehat{x}}+\left[\left(\bar{u}_{j}^{n}\right)^{p-1} \cdot\left(\bar{u}_{j}^{n}\right)\right]_{\widehat{x}}\right\} .
$$

By the definition of $\left(u_{j}^{n}\right)_{\hat{t}}$, it follows from the first term of (20) that

$$
h \sum_{j=1}^{J-1}\left(\frac{1}{2 \tau}\left(u_{j}^{n+1}-u_{j}^{n-1}\right) \cdot 2 \bar{u}_{j}^{n}\right)=\frac{1}{2 \tau}\left(\left\|u^{n+1}\right\|^{2}-\left\|u^{n-1}\right\|^{2}\right) .
$$

By the definition of $u_{\widehat{x}},(14)$, and Lemma 1 , it follows from the second and the third term of (20) that

$$
\begin{gathered}
\sum_{j=1}^{J-1}\left(\left(\bar{u}_{j}^{n}\right)_{x}+\left(\bar{u}_{j}^{n}\right)_{\bar{x}}\right) \cdot \bar{u}_{j}^{n}=\sum_{j=1}^{J-1}\left(\bar{u}_{j}^{n}\right)_{x} \cdot \bar{u}_{j}^{n}+\sum_{j=1}^{J-1}\left(\bar{u}_{j}^{n}\right)_{\bar{x}} \cdot \bar{u}_{j}^{n}=0, \\
\sum_{j=1}^{J-1}\left(\bar{u}_{j}^{n}\right)_{x \bar{x} \hat{x}} \cdot 2 \bar{u}_{j}^{n}=0 .
\end{gathered}
$$

According to the boundary condition (12) and (14) of Lemma 1, it follows from the forth term that

$$
\begin{gathered}
h \sum_{j=1}^{J-1} \frac{1}{2 \tau}\left(\left(u_{j}^{n+1}\right)_{x x \bar{x} \bar{x}}-\left(u_{j}^{n-1}\right)_{x x \bar{x} \bar{x}}\right) \cdot 2 \bar{u}_{j}^{n} \\
=\frac{1}{2 \tau}\left(\left\|u_{x x}^{n+1}\right\|^{2}-\left\|u_{x x}^{n-1}\right\|^{2}\right) .
\end{gathered}
$$

According to (13) and (14), we have

$$
\begin{aligned}
\left\langle P, 2 \bar{u}^{n}\right\rangle= & \frac{2 p h}{p+1} \sum_{j=1}^{J-1}\left[\left(u_{j}^{n}\right)^{p-1} \cdot\left(\bar{u}_{j}^{n}\right)_{\widehat{x}}+\left[\left(u_{j}^{n}\right)^{p-1} \bar{u}_{j}^{n}\right]_{\widehat{x}}\right] \cdot \bar{u}_{j}^{n} \\
= & \frac{p}{p+1} \sum_{j=1}^{J-1}\left[\left(u_{j}^{n}\right)^{p-1} \cdot\left(\bar{u}_{j+1}^{n}-\bar{u}_{j-1}^{n}\right)\right. \\
& \left.+\left(u_{j+1}^{n}\right)^{p-1} \bar{u}_{j+1}^{n}-\left(u_{j-1}^{n}\right)^{p-1} \bar{u}_{j-1}^{n}\right] \bar{u}_{j}^{n} \\
= & \frac{p}{p+1} \sum_{j=1}^{J-1}\left[\left(u_{j}^{n}\right)^{p-1} \bar{u}_{j+1}^{n} \bar{u}_{j}^{n}-\left(u_{j+1}^{n}\right)^{p-1} \bar{u}_{j+1}^{n} \bar{u}_{j}^{n}\right] \\
& -\frac{p}{p+1} \sum_{j=1}^{J-1}\left[\left(u_{j-1}^{n}\right)^{p-1} \bar{u}_{j}^{n} \bar{u}_{j-1}^{n}-\left(u_{j}^{n}\right)^{p-1} \bar{u}_{j}^{n} \bar{u}_{j-1}^{n}\right] \\
= & 0 .
\end{aligned}
$$

Substituting (22)-(25) into (20), we have

$$
\left(\left\|u^{n+1}\right\|^{2}-\left\|u^{n-1}\right\|^{2}\right)+\left(\left\|u_{x x}^{n+1}\right\|^{2}-\left\|u_{x x}^{n-1}\right\|^{2}\right)=0 .
$$

By the definition of $E^{n}$, (19) holds. It implies that the difference scheme is conservative for energy.

In order to prove the boundedness of the numerical solution, we introduce the following lemma [23].

Lemma 4 (Discrete Sobolev's inequality). There exist two constants $C_{1}$ and $C_{2}$ such that

$$
\left\|u^{n}\right\|_{\infty} \leq C_{1}\left\|u^{n}\right\|+C_{2}\left\|u_{x}^{n}\right\|
$$

Theorem 5. Suppose $u_{0} \in H_{0}^{2}\left[X_{l}, X_{r}\right]$; then the solution $u^{n}$ of (10)-(12) satisfies $\left\|u^{n}\right\| \leq C,\left\|u_{x}^{n}\right\| \leq C$, which yield $\left\|u^{n}\right\|_{\infty} \leq$ $C(n=1,2, \ldots, N)$.

Proof. It follows from (19) that

$$
\left\|u^{n}\right\| \leq C, \quad\left\|u_{x x}^{n}\right\| \leq C .
$$

By Lemma 1 and Schwartz inequality, we get

$$
\left\|u_{x}^{n}\right\|^{2} \leq\left\|u^{n}\right\|\left\|u_{x x}^{n}\right\| \leq \frac{1}{2}\left(\left\|u^{n}\right\|+\left\|u_{x x}^{n}\right\|\right) \leq C .
$$

According to Lemma 4 , we have $\left\|u^{n}\right\|_{\infty} \leq C(n=1,2, \ldots, N)$. 


\section{Solvability}

Theorem 6. There exists $u^{n} \in Z_{h}^{0}(1 \leq n \leq N)$ which satisfies the difference scheme (10)-(12).

Proof. By mathematical induction, it is obvious that $u^{0}$ is uniquely determined by the initial condition (11). We can choose a second-order method to compute $u^{1}$ (such as C$\mathrm{N}$ scheme $[10,15])$. It implies that $u^{0}, u^{1}$ are uniquely determined. Now assuming $u^{0}, u^{1}, \ldots, u^{n}$ are uniquely solvable, consider $u^{n+1}$ in (10) which satisfies

$$
\begin{aligned}
& \frac{1}{2 \tau} u_{j}^{n+1}+\left(u_{j}^{n+1}\right)_{\widehat{x}}+\left(u_{j}^{n+1}\right)_{x \bar{x} \hat{x}}+\frac{1}{2 \tau}\left(u_{j}^{n+1}\right)_{x x \bar{x} \bar{x}} \\
& +\frac{p}{2(p+1)}\left\{\left(u_{j}^{n}\right)^{p-1}\left(u_{j}^{n+1}\right)_{\widehat{x}}+\left[\left(u_{j}^{n}\right)^{p-1} u_{j}^{n+1}\right]_{\widehat{x}}\right\}=0 .
\end{aligned}
$$

Computing the inner product of (30) with $u^{n+1}$, by (23) and (24), we obtain

$$
\frac{1}{2 \tau}\left\|u^{n+1}\right\|^{2}+\frac{1}{2 \tau}\left\|u_{x x}^{n+1}\right\|^{2}+\left\langle\Phi\left(u^{n}, u^{n+1}\right), u^{n+1}\right\rangle=0,
$$

where $\Phi\left(u^{n}, u^{n+1}\right)=(p / 2(p+1))\left\{\left(u^{n}\right)^{p-1}\left(u^{n+1}\right)_{\widehat{x}}+\right.$ $\left.\left[\left(u^{n}\right)^{p-1} u^{n+1}\right]_{\widehat{x}}\right\}$,

$$
\begin{aligned}
& \left\langle\Phi\left(u^{n}, u^{n+1}\right), u^{n+1}\right\rangle \\
& =\frac{p h}{2(p+1)} \sum_{j=1}^{J-1}\left\{\left(u_{j}^{n}\right)^{p-1}\left(u_{j}^{n+1}\right)_{\widehat{x}}+\left[\left(u_{j}^{n}\right)^{p-1} u_{j}^{n+1}\right]_{\widehat{x}}\right\} u_{j}^{n+1} \\
& =\frac{p}{4(p+1)} \sum_{j=1}^{J-1}\left\{\left(u_{j}^{n}\right)^{p-1}\left(u_{j+1}^{n+1}-u_{j-1}^{n+1}\right)\right. \\
& \left.\quad+\left[\left(u_{j+1}^{n}\right)^{p-1} u_{j+1}^{n+1}-\left(u_{j-1}^{n}\right)^{p-1} u_{j-1}^{n+1}\right]\right\} u_{j}^{n+1}
\end{aligned}
$$

$=0$.

It follows from (31) that

$$
\frac{1}{2 \tau}\left\|u^{n+1}\right\|^{2}+\frac{1}{2 \tau}\left\|u_{x x}^{n+1}\right\|^{2}=0 .
$$

That is, there uniquely exists trivial solution satisfying (30). Therefore, $u_{j}^{n+1}$ in (10) is uniquely solvable.

This completes the proof of Theorem 6 .

\section{The Convergence and Stability of the Scheme}

As usual, in order to prove the convergence and stability of the average linear difference scheme, we need to introduce the Discrete Gronwall inequality [23].

Lemma 7. Suppose $w(k), \rho(k)$ are nonnegative mesh functions and $\rho(k)$ is nondecreasing. If $C>0$ and

$$
w(k) \leq \rho(k)+C \tau \sum_{l=0}^{k-1} w(l), \quad \forall k,
$$

then

$$
w(k) \leq \rho(k) e^{C \tau k}, \quad \forall k .
$$

Now, we discuss the convergence of the scheme (10)-(12); let $v_{j}^{n}=v\left(x_{j}, t_{n}\right)$ be the analytical solution of problem (3)-(5); then the truncation error of the scheme (10)-(12) is

$$
\begin{aligned}
R_{j}^{n}= & \left(v_{j}^{n}\right)_{\widehat{t}}+\left(v_{j}^{n}\right)_{\widehat{x}}+\left(v_{j}^{n}\right)_{x \bar{x} \hat{x}}+\left(v_{j}^{n}\right)_{x x \bar{x} \bar{x} \hat{t}} \\
& +\frac{p}{1+p}\left\{\left(v_{j}^{n}\right)^{p-1}\left(\bar{v}_{j}^{n}\right)_{\widehat{x}}+\left[\left(v_{j}^{n}\right)^{p-1}\left(\bar{v}_{j}^{n}\right)\right]_{\widehat{x}}\right\} .
\end{aligned}
$$

Using Taylor expansion, we know that $R_{j}^{n}=O\left(\tau^{2}+h^{2}\right)$ holds if $\tau, h \rightarrow 0$.

Theorem 8. Supposing $u_{0} \in H_{0}^{2}\left[X_{l}, X_{r}\right], u(x, t) \in C^{5,3}$, then the solution $u^{n}$ of the scheme (10)-(12) converges to the solution of problem (3)-(5) and the rate of convergence is $R_{j}^{n}=O\left(\tau^{2}+\right.$ $h^{2}$ ) by the $\|\cdot\|_{\infty}$ norm.

Proof. Subtracting (10) from (36) and letting $e_{j}^{n}=v_{j}^{n}-u_{j}^{n}$, we have

$$
\begin{aligned}
R_{j}^{n}= & \left(e_{j}^{n}\right)_{\widehat{t}}+\left(\bar{e}_{j}^{n}\right)_{\widehat{x}}+\left(\bar{e}_{j}^{n}\right)_{x \bar{x} \hat{x}}+\left(e_{j}^{n}\right)_{x x \bar{x} \bar{x} \hat{t}} \\
& +\frac{p}{1+p}\left\{\left(v_{j}^{n}\right)^{p-1}\left(\bar{v}_{j}^{n}\right)_{\widehat{x}}+\left[\left(v_{j}^{n}\right)^{p-1} \bar{v}_{j}^{n}\right]_{\widehat{x}}\right\} \\
& -\frac{p}{1+p}\left\{\left(u_{j}^{n}\right)^{p-1}\left(\bar{u}_{j}^{n}\right)_{\widehat{x}}+\left[\left(u_{j}^{n}\right)^{p-1} \bar{u}_{j}^{n}\right]_{\widehat{x}}\right\} .
\end{aligned}
$$

Computing the inner product of (37) with $2 e^{-n}$ and using

$$
\left\langle\left(\bar{e}^{n}\right)_{\widehat{x}}, 2 e^{n}\right\rangle=0, \quad\left\langle\left(e^{n}\right)_{x \bar{x} \widehat{x}}, 2 e^{n}\right\rangle=0 .
$$

Similar to (22) and (24), we get

$$
\begin{aligned}
\left\langle R^{n}, 2 e^{n}\right\rangle= & \frac{1}{2 \tau}\left(\left\|e^{n+1}\right\|^{2}-\left\|e^{n-1}\right\|^{2}\right) \\
& +\frac{\gamma}{2 \tau}\left(\left\|e_{x x}^{n+1}\right\|^{2}-\left\|e_{x x}^{n-1}\right\|^{2}\right)+\left\langle Q_{1}+Q_{2}, 2 \bar{e}^{n}\right\rangle,
\end{aligned}
$$

where

$$
\begin{aligned}
Q_{1} & =\frac{p}{1+p}\left[\left(v^{n}\right)^{p-1}\left(v^{n}\right)_{\widehat{x}}-\left(u^{n}\right)^{p-1}\left(\bar{u}^{n}\right)_{\widehat{x}}\right], \\
Q_{2} & =\frac{p}{1+p}\left\{\left[\left(v^{n}\right)^{p-1} \bar{v}^{n}\right]_{\widehat{x}}-\left[\left(u^{n}\right)^{p-1} \bar{u}^{n}\right]_{\widehat{x}}\right\} .
\end{aligned}
$$

Therefore, we get

$$
\begin{gathered}
\left(\left\|e^{n+1}\right\|^{2}-\left\|e^{n-1}\right\|^{2}\right)+\left(\left\|e_{x x}^{n+1}\right\|^{2}-\left\|e_{x x}^{n-1}\right\|^{2}\right) \\
=2 \tau\left\langle r^{n}, 2 e^{n}\right\rangle-2 \tau\left\langle Q_{1}+Q_{2}, 2 \bar{e}^{n}\right\rangle .
\end{gathered}
$$


According to Lemma 2, Theorem 5, and Schwartz inequality, we have

$$
\begin{aligned}
-\left\langle Q_{1}, 2 \bar{e}^{n}\right\rangle= & \frac{-2 p}{1+p} h \sum_{j=0}^{J-1}\left[\left(v_{j}^{n}\right)^{p-1}\left(\bar{v}_{j}^{n}\right)_{\widehat{x}}-\left(u_{j}^{n}\right)^{p-1}\left(\bar{u}_{j}^{n}\right)_{\widehat{x}}\right] \bar{e}_{j}^{n} \\
= & \frac{-2 p}{1+p} h \sum_{j=0}^{J-1}\left(v_{j}^{n}\right)^{p-1}\left(\bar{e}_{j}^{n}\right)_{\widehat{x}} \bar{e}_{j}^{n} \\
& +\frac{-2 p}{1+p} h \sum_{j=0}^{J-1}\left[\left(v_{j}^{n}\right)^{p-1}-\left(u_{j}^{n}\right)^{p-1}\right]\left(\bar{u}_{j}^{n}\right)_{\widehat{x}} \bar{e}_{j}^{n} \\
= & \frac{-2 p}{1+p} h \sum_{j=0}^{J-1}\left(v_{j}^{n}\right)^{p-1}\left(\bar{e}_{j}^{n}\right)_{\widehat{x}} \bar{e}_{j}^{n} \\
& +\frac{-2 p}{1+p} h \sum_{j=0}^{J-1}\left[e_{j}^{n} \sum_{k=0}^{p-2}\left(v_{j}^{n}\right)^{p-2-k}\left(u_{j}^{n}\right)^{k}\right]\left(\bar{u}_{j}^{n}\right)_{\widehat{x}} \bar{e}_{j}^{n} \\
\leq & C h \sum_{j=0}^{J-1}\left|\left(\bar{e}_{j}^{n}\right)_{\widehat{x}}\right| \cdot\left|\bar{e}_{j}^{n}\right|+C h \sum_{j=0}^{J-1}\left|\left(e_{j}^{n}\right) \|\left(\bar{e}_{j}^{n}\right)\right| \\
\leq & C\left[\left\|\bar{e}_{x}^{n}\right\|^{2}+\left\|e^{n}\right\|^{2}+\left\|\bar{e}^{n}\right\|^{2}\right] \\
\leq & C\left[\left\|e_{x}^{n+1}\right\|^{2}+\left\|e_{x}^{n-1}\right\|^{2}+\left\|e^{n+1}\right\|^{2}+\left\|e^{n}\right\|^{2}\right. \\
& \left.+\left\|e^{n-1}\right\|^{2}\right] .
\end{aligned}
$$

Similarly,

$$
\begin{gathered}
-\left\langle Q_{2}, 2 \bar{e}^{n}\right\rangle \leq C\left[\left\|e_{x}^{n+1}\right\|^{2}+\left\|e_{x}^{n-1}\right\|^{2}+\left\|e^{n+1}\right\|^{2}+\left\|e^{n}\right\|^{2}\right. \\
\left.+\left\|e^{n-1}\right\|^{2}\right] .
\end{gathered}
$$

Furthermore,

$$
\begin{aligned}
\left\langle R^{n}, 2 \bar{e}^{n}\right\rangle & =\left\langle R^{n}, e^{n+1}+e^{n-1}\right\rangle \\
& \leq\left\|R^{n}\right\|^{2}+\frac{1}{2}\left[\left\|e^{n+1}\right\|^{2}+\left\|e^{n-1}\right\|^{2}\right] .
\end{aligned}
$$

Substituting (42)-(44) into (41), we get

$$
\begin{aligned}
\left(\left\|e^{n+1}\right\|^{2}-\left\|e^{n-1}\right\|^{2}\right)+\left(\left\|e_{x x}^{n+1}\right\|^{2}-\left\|e_{x x}^{n-1}\right\|^{2}\right) & \\
\leq C \tau & {\left[\left\|e_{x}^{n+1}\right\|^{2}+\left\|e_{x}^{n}\right\|^{2}+\left\|e_{x}^{n-1}\right\|^{2}\right.} \\
& \left.+\left\|e^{n+1}\right\|^{2}+\left\|e^{n}\right\|^{2}+\left\|e^{n-1}\right\|^{2}\right]+2 \tau\left\|R^{n}\right\|^{2} .
\end{aligned}
$$

Similar to the proof of (29)

$$
\left\|e_{x}^{n}\right\|^{2} \leq\left\|e^{n}\right\|\left\|e_{x x}^{n}\right\| \leq \frac{1}{2}\left(\left\|e^{n}\right\|+\left\|e_{x x}^{n}\right\|\right) \leq C .
$$

It follows from (45) that

$$
\begin{gathered}
{\left[\left(\left\|e^{n+1}\right\|^{2}+\left\|e^{n}\right\|^{2}\right)+\gamma\left(\left\|e_{x x}^{n+1}\right\|^{2}+\left\|e_{x x}^{n}\right\|^{2}\right)\right]} \\
-\left[\left(\left\|e^{n}\right\|^{2}+\left\|e^{n-1}\right\|^{2}\right)+\gamma\left(\left\|e_{x x}^{n}\right\|^{2}+\left\|e_{x x}^{n-1}\right\|^{2}\right)\right] \\
\leq C \tau\left[\left\|e^{n+1}\right\|^{2}+\left\|e^{n}\right\|^{2}+\left\|e^{n-1}\right\|^{2}+\left\|e_{x x}^{n+1}\right\|^{2}\right. \\
\left.+\left\|e_{x x}^{n}\right\|^{2}+\left\|e_{x x}^{n-1}\right\|^{2}\right]+2 \tau\left\|R^{n}\right\|^{2} .
\end{gathered}
$$

Let $B^{n}=\left\|e^{n}\right\|^{2}+\left\|e^{n+1}\right\|^{2}+\left\|e_{x x}^{n}\right\|^{2}+\left\|e_{x x}^{n+1}\right\|^{2}$; it follows from (47) that

$$
(1-C \tau)\left(B^{n+1}-B^{n}\right) \leq 2 C \tau B^{n}+2 \tau\left\|R^{n}\right\|^{2} .
$$

If $\tau$ is sufficiently small which satisfies $1-C \tau>0$, we get

$$
B^{n+1}-B^{n} \leq C \tau B^{n}+C \tau\left\|R^{n}\right\|^{2} .
$$

Summing up (49) from 0 to $n-1$, we get

$$
B^{n} \leq B^{0}+C \tau \sum_{l=0}^{n-1} B^{n}+C \tau \sum_{l=0}^{n-1}\left\|R^{l}\right\|^{2} .
$$

Choose a second-order method to compute $u^{1}$ (such as C-N scheme) and notice that

$$
\tau \sum_{l=0}^{n-1}\left\|R^{l}\right\|^{2} \leq n \tau \max _{0 \leq l \leq n-1}\left\|R^{l}\right\|^{2} \leq T \cdot O\left(\tau^{2}+h^{2}\right)^{2} .
$$

From the discrete initial conditions, we know that $e^{0}=0$; then we have

$$
B^{0}=O\left(\tau^{2}+h^{2}\right)^{2}
$$

Therefore,

$$
B^{n} \leq O\left(\tau^{2}+h^{2}\right)^{2}+C \tau \sum_{l=0}^{n-1} B^{l} .
$$

According to Lemma 7, we get

$$
B^{n} \leq O\left(\tau^{2}+h^{2}\right)^{2} .
$$

It implies

$$
\left\|e^{n}\right\| \leq O\left(\tau^{2}+h^{2}\right), \quad\left\|e_{x x}^{n}\right\| \leq O\left(\tau^{2}+h^{2}\right) .
$$

It follows from (29) that

$$
\left\|e_{x}^{n}\right\| \leq O\left(\tau^{2}+h^{2}\right) .
$$

By Lemma 4, we have

$$
\left\|e^{n}\right\|_{\infty} \leq O\left(\tau^{2}+h^{2}\right) .
$$

This completes the proof of Theorem 8 . 


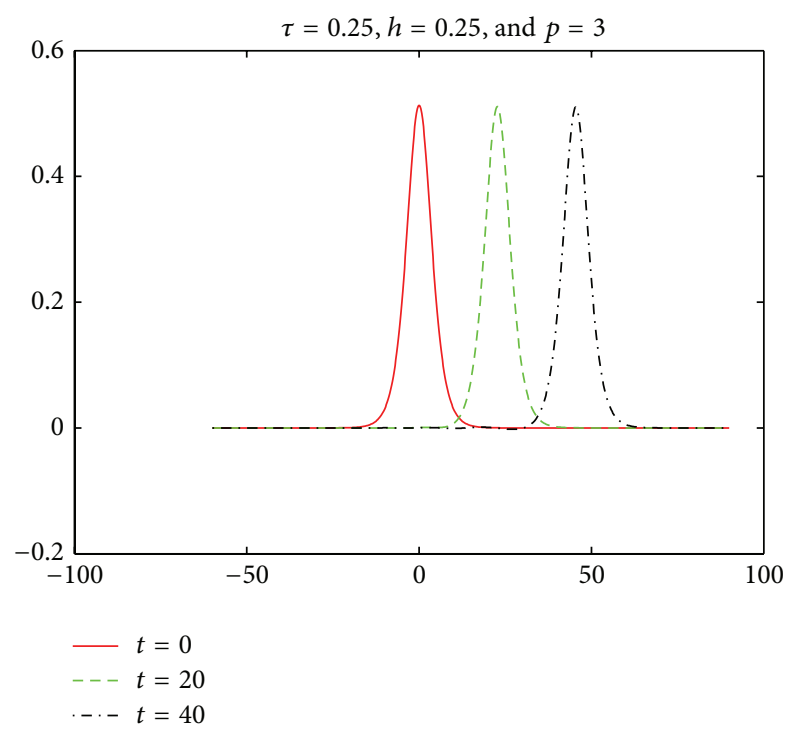

FigURE 1: Wave graph of $u(x, t)$ at various times when $p=3$ and $\tau=h=0.25$.

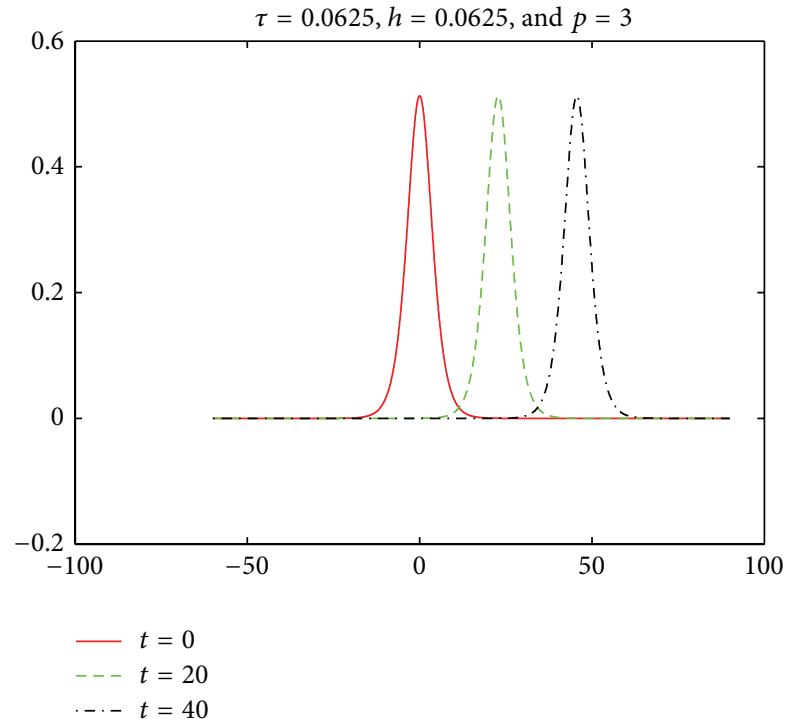

FIGURE 2: Wave graph of $u(x, t)$ at various times when $p=3$ and $\tau=h=0.0625$.

In order to prove the stability of the difference scheme, we import the initial-boundary problem

$$
\begin{gathered}
u_{t}+u_{x}+u_{x x x}+u_{x x x x t}+\left(u^{p}\right)_{x}=\omega(x, t), \\
u\left(X_{l}, t\right)=u\left(X_{r}, t\right)=0, \\
u_{x}\left(X_{l}, t\right)=u_{x}\left(X_{r}, t\right)=0, \\
u_{x x}\left(X_{l}, t\right)=u_{x x}\left(X_{r}, t\right)=0, \\
t \in[0, T], \\
u(x, 0)=u_{0}(x)+\psi(x), \quad x \in\left[X_{l}, X_{r}\right],
\end{gathered}
$$

where $\omega(x, t), \psi(x)$ are smooth enough.

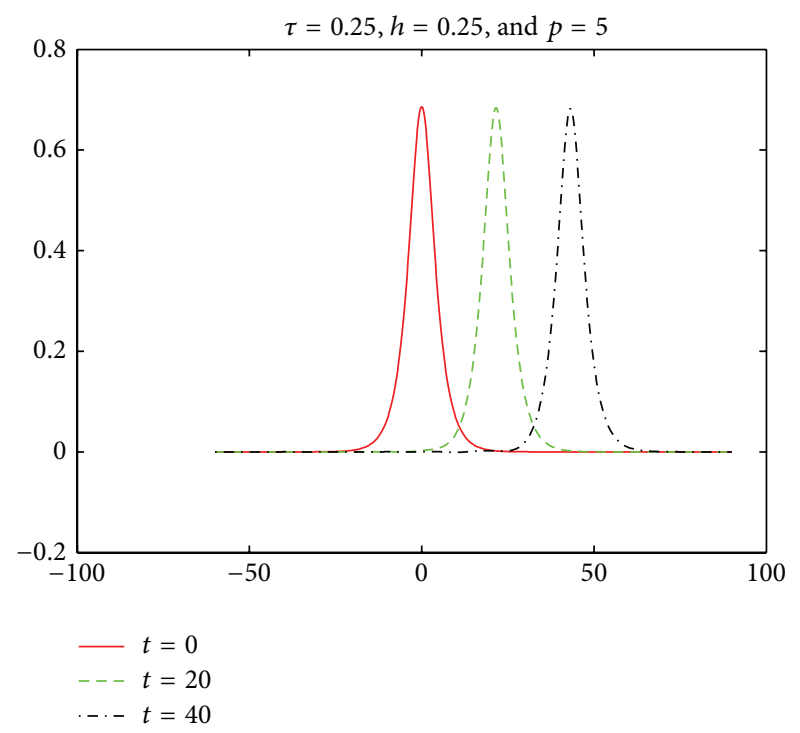

FigURE 3: Wave graph of $u(x, t)$ at various times when $p=5$ and $\tau=h=0.25$.

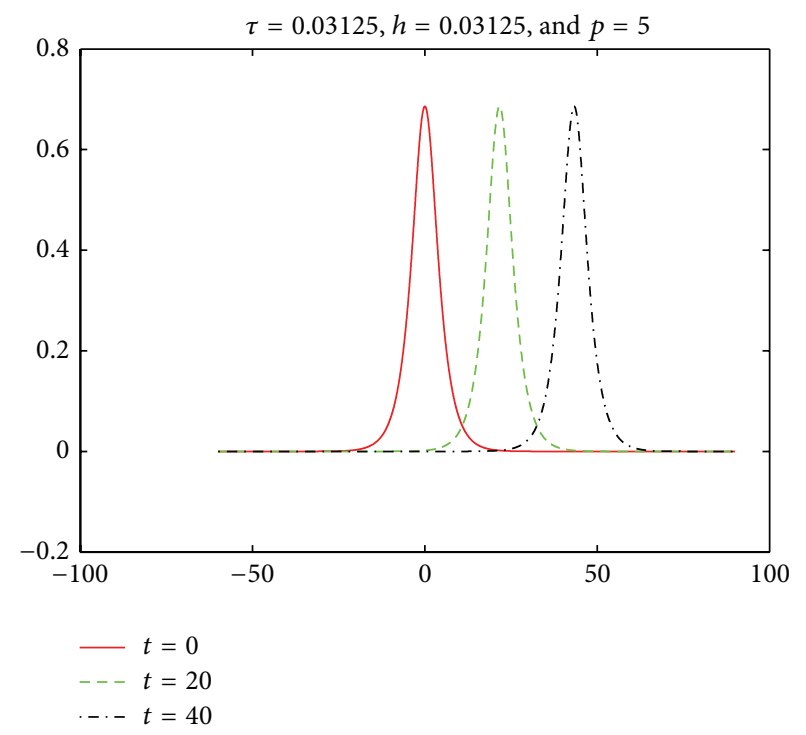

FIgURE 4: Wave graph of $u(x, t)$ at various times when $p=5$ and $\tau=h=0.03125$.

We propose the difference scheme of the problem (58)

$$
\begin{aligned}
\left(U_{j}^{n}\right)_{\hat{t}} & +\left(\bar{U}_{j}^{n}\right)_{\widehat{x}}+\left(\bar{U}_{j}^{n}\right)_{x \bar{x} \widehat{x}}+\left(U_{j}^{n}\right)_{x x \bar{x} \bar{x} \hat{t}} \\
& +\frac{p}{1+p}\left\{\left(U_{j}^{n}\right)^{p-1}\left(\bar{U}_{j}^{n}\right)_{\widehat{x}}+\left[\left(U_{j}^{n}\right)^{p-1} \bar{U}_{j}^{n}\right]_{\widehat{x}}\right\}+\left(\omega_{j}^{n}\right)=0,
\end{aligned}
$$$$
U_{j}^{0}=U_{0}\left(x_{j}\right)+\psi_{j}, \quad 0 \leq j \leq J-1,
$$ 
TABle 1: The errors estimated in the sense of $L_{\infty}$ for $p=3$ at $T=40$.

\begin{tabular}{lccc}
\hline$h$ & $\tau$ & $\left\|e^{N}\right\|_{\infty}$ & $\left\|e^{N}(h, \tau)\right\|_{\infty} /\left\|e^{2 N}(h / 2, \tau / 2)\right\|_{\infty}$ \\
\hline 0.25 & 0.25 & $1.34986 e-002$ & - \\
0.125 & 0.125 & $3.42489 e-003$ & 3.94134 \\
0.0625 & 0.0625 & $8.59570 e-004$ & 3.98441 \\
0.03125 & 0.03125 & $2.15150 e-004$ & 3.99521 \\
\hline
\end{tabular}

TABLE 2: The errors estimated in the sense of $L_{\infty}$ for $p=5$ at $T=40$.

\begin{tabular}{lccc}
\hline$h$ & $\tau$ & $\left\|e^{N}\right\|_{\infty}$ & $\left\|e^{N}(h, \tau)\right\|_{\infty} /\left\|e^{2 N}(h / 2, \tau / 2)\right\|_{\infty}$ \\
\hline 0.25 & 0.25 & $1.79985 e-002$ & - \\
0.125 & 0.125 & $4.56804 e-003$ & 3.94009 \\
0.0625 & 0.0625 & $1.14689 e-003$ & 3.98299 \\
0.03125 & 0.03125 & $2.87085 e-004$ & 3.99494 \\
\hline
\end{tabular}

$$
\begin{gathered}
U_{0}^{n}=U_{J}^{n}=0, \quad\left(U_{0}^{n}\right)_{\widehat{x}}=\left(U_{J}^{n}\right)_{\widehat{x}}=0, \\
\left(U_{0}^{n}\right)_{x \bar{x}}=\left(U_{J}^{n}\right)_{\bar{x} x}=0,
\end{gathered}
$$

where $\omega_{j}^{n}=\omega\left(x_{j}, t_{n}\right), \psi_{j}=\psi\left(x_{j}\right)$.

Similar to the proof of Theorem 8 , we can prove the stability Theorem 9.

Theorem 9. Supposing $\left\{u_{j}^{n}\right\}$ is the solution of the scheme (10)(12) and $\left\{U_{j}^{n}\right\}$ is the solution of the scheme (59), denote $\varepsilon_{j}^{n}=$ $U_{j}^{n}-u_{j}^{n}$. If the mesh steps $h, \tau$ are small enough, we can get the stability result

$$
\left\|\varepsilon^{n}\right\|+\left\|\varepsilon_{x x}^{n}\right\| \leq C\left(\|\psi\|^{2}+\tau \sum_{l=0}^{n-1}\left\|\omega^{l}\right\|^{2}\right)
$$

\section{Numerical Validation}

In this section, we conduct some numerical experiments to verify theoretical results obtained in the previous sections. We take $X_{l}=-60, X_{r}=90$ and consider the two cases $p=3,5$, respectively.

According to $[16,17]$, when $p=3$, the soliton solution is as follows:

$$
\begin{aligned}
u(x, t)= & \frac{1}{4} \sqrt{-15+3 \sqrt{41}} \\
& \times \operatorname{sech}^{2} \frac{1}{4} \sqrt{\frac{-5+\sqrt{41}}{2}}\left[x-\frac{1}{10}(5+\sqrt{41}) t\right],
\end{aligned}
$$

and the initial condition is

$$
u(x, 0)=\frac{1}{4} \sqrt{-15+3 \sqrt{41}} \operatorname{sech}^{2} \frac{1}{4} \sqrt{\frac{-5+\sqrt{41}}{2}} x .
$$

When $p=5$, the soliton solution is

$$
\begin{aligned}
u(x, t)= & \sqrt[4]{\frac{4}{15}(-5+\sqrt{34})} \\
& \times \operatorname{sech} \frac{1}{3} \sqrt{-5+\sqrt{34}}\left[x-\frac{1}{10}(5+\sqrt{34}) t\right],
\end{aligned}
$$

and initial condition is

$$
u(x, 0)=\sqrt[4]{\frac{4}{15}(-5+\sqrt{34})} \operatorname{sech} \frac{1}{3} \sqrt{-5+\sqrt{34}} x .
$$

Since the three-level implicit finite difference scheme cannot start by itself, we need to select other two-level schemes (such as the C-N Scheme) to get $u^{1}$. Then, reusing initial value $u^{0}$, we apply the average implicit linear threelevel difference scheme (10)-(12) for the problem (4)-(6) to work out $u^{2}, u^{3}, \ldots$ Iterative method is not required for the linear scheme, so it saves computing time.

First of all, we simulate the wave graph of the numerical solution to the average linear implicit scheme (10)-(12). The wave graph comparison of numerical solution $u\left(x_{j}, t_{n}\right)$ between different time step and space step at various times is given in Figures 1, 2, 3, and 4 for $p=3$ and $p=5$. The figures show that the height of the wave graph at different time is almost identical. It implies that the energy is conservative.

Secondly, we conduct numerical simulations in different time step and space step for $p=3$ and $p=5$, respectively, when time is $40 \mathrm{~s}$. We list some results in Tables 1 and 2 for $p=$ 3 and $p=5$, respectively. All results show that the numerical solution is 2nd-order convergent and unconditionally stable. Meanwhile, we also list the conservative invariants $E^{n}$ at different time in Tables 3 and 4 for $p=3$ and $p=5$. These results testify that the studied scheme is conservative for energy.

\section{Conclusions}

In brief, we first proposed an average linear implicit scheme for the Generalized Rosenau-KdV equation, which has a wide range of applications in various areas of scientific researches. The solvability, convergence, energy conservation, 
TABLE 3: The energy of different time in different time step and space step for $p=3$.

\begin{tabular}{lcccc}
\hline$(h, \tau)$ & $T=10 \mathrm{~s}$ & $T=20 \mathrm{~s}$ & $T=30 \mathrm{~s}$ & $T=40 \mathrm{~s}$ \\
\hline$(0.25,0.25)$ & 1.682528993311382 & 1.682528993311723 & 1.682528993311437 & 1.682528993311429 \\
$(0.125,0.125)$ & 1.682543082559648 & 1.682543082567992 & 1.682543082564207 & 1.682543082565549 \\
$(0.0625,0.0625)$ & 1.682546611032036 & 1.682546611133070 & 1.682546611138651 & 1.682546611129625 \\
$(0.03125,0.03125)$ & 1.682547494622366 & 1.682547493895496 & 1.682547493110830 & 1.682547493646520 \\
\hline
\end{tabular}

TABLE 4: The energy of different time in different time step and space step for $p=5$.

\begin{tabular}{lcccc}
\hline$(h, \tau)$ & $T=10 \mathrm{~s}$ & $T=20 \mathrm{~s}$ & $T=30 \mathrm{~s}$ & $T=40 \mathrm{~s}$ \\
\hline$(0.25,0.25)$ & 3.110674902410195 & 3.110674902410293 & 3.110674902409525 & 3.110674902410005 \\
$(0.125,0.125)$ & 3.110702938793859 & 3.110702938807032 & 3.110702938804688 & 3.110702938809395 \\
$(0.0625,0.0625)$ & 3.110709964290841 & 3.110709964308914 & 3.110709964158443 & 3.110709964168867 \\
$(0.03125,0.03125)$ & 3.110711721444649 & 3.110711720432934 & 3.110711717170060 & 3.110711718996421 \\
\hline
\end{tabular}

and stability with $O\left(\tau^{2}+h^{2}\right)$ of the discrete solutions were analyzed in detail. Numerical simulations were carried out to testify that the theoretical analyses are right and our scheme is accurate and reliable.

\section{Conflict of Interests}

The authors declare that there is no conflict of interests regarding the publication of this paper.

\section{References}

[1] Y. Cui and D. K. Mao, "Numerical method satisfying the first two conservation laws for the Korteweg-de Vries equation," Journal of Computational Physics, vol. 227, no. 1, pp. 376-399, 2007.

[2] S. Zhu and J. Zhao, "The alternating segment explicit-implicit scheme for the dispersive equation," Applied Mathematics Letters, vol. 14, no. 6, pp. 657-662, 2001.

[3] A. R. Bahadır, "Exponential finite-difference method applied to Korteweg-de Vries equation for small times," Applied Mathematics and Computation, vol. 160, no. 3, pp. 675-682, 2005.

[4] S. Özer and S. Kutluay, "An analytical-numerical method for solving the Korteweg-de Vries equation," Applied Mathematics and Computation, vol. 164, no. 3, pp. 789-797, 2005.

[5] P. Rosenau, "A quasi-continuous description of a nonlinear transmission line," Physica Scripta, vol. 34, pp. 827-829, 1986.

[6] P. Rosenau, "Dynamics of dense discrete systems," Progress of Theoretical Physics, vol. 79, pp. 1028-1042, 1988.

[7] M. A. Park, "On the Rosenau equation," Matemática Aplicada e Computacional, vol. 9, no. 2, pp. 145-152, 1990.

[8] S. K. Chung and S. N. Ha, "Finite element Galerkin solutions for the Rosenau equation," Applicable Analysis, vol. 54, no. 1-2, pp. 39-56, 1994.

[9] K. Omrani, F. Abidi, T. Achouri, and N. Khiari, "A new conservative finite difference scheme for the Rosenau equation," Applied Mathematics and Computation, vol. 201, no. 1-2, pp. 3543, 2008 .
[10] S. K. Chung, "Finite difference approximate solutions for the Rosenau equation," Applicable Analysis, vol. 69, no. 1-2, pp. 149156, 1998.

[11] S. K. Chung and A. K. Pani, "Numerical methods for the Rosenau equation," Applicable Analysis, vol. 77, no. 3-4, pp. 351369, 2001.

[12] S. A. V. Manickam, A. K. Pani, and S. K. Chung, "A second-order splitting combined with orthogonal cubic spline collocation method for the Rosenau equation," Numerical Methods for Partial Differential Equations, vol. 14, no. 6, pp. 695-716, 1998.

[13] Y. D. Kim and H. Y. Lee, "The convergence of finite element Galerkin solution for the Roseneau equation," The Korean Journal of Computational \& Applied Mathematics, vol. 5, no. 1, pp. 171-180, 1998.

[14] J. M. Zuo, "Solitons and periodic solutions for the Rosenau$\mathrm{KdV}$ and Rosenau-Kawahara equations," Applied Mathematics and Computation, vol. 215, no. 2, pp. 835-840, 2009.

[15] J. Hu, Y. Xu, and B. Hu, "Conservative linear difference scheme for Rosenau-KdV equation," Advances in Mathematical Physics, vol. 2013, Article ID 423718, 7 pages, 2013.

[16] A. Esfahani, "Solitary wave solutions for generalized RosenauKdV equation," Communications in Theoretical Physics, vol. 55, no. 3, pp. 396-398, 2011.

[17] P. Razborova, H. Triki, and A. Biswas, "Perturbation of dispersive shallow water waves," Ocean Engineering, vol. 63, pp. 1-7, 2013.

[18] G. Ebadi, A. Mojaver, H. Triki, A. Yildirim, and A. Biswas, "Topological solitons and other solutions of the Rosenau-KdV equation with power law nonlinearity," Romanian Journal of Physics, vol. 58, no. 1-2, pp. 3-14, 2013.

[19] A. Saha, "Topological 1-soliton solutions for the generalized Rosenau-KdV equation," Fundamental Journal of Mathematical Physics, vol. 2, no. 1, pp. 19-23, 2012.

[20] A. Chowdhury and A. Biswas, "Singular solitons and numerical analysis of $\Phi$-four equation," Mathematical Sciences, vol. 6, article 42, 2012.

[21] P. Suarez, S. Johnson, and A. Biswas, "Chebyshev split-step scheme for the sine-Gordon equation in $2+1$ dimensions," International Journal of Nonlinear Sciences and Numerical Simulation, vol. 14, no. 1, pp. 69-75, 2013. 
[22] M. A. Christou, "Christov-galerkin expansion for localized solutions in model equations with higher order dispersion," in Proceedings of the 33rd International Conference on Applications of Mathematics in Engineering and Economics, M. D. Todorov, Ed., CP946, pp. 91-98, June 2007.

[23] Y. L. Zhou, Applications of Discrete Functional Analysis to the Finite Difference Method, International Academic Publishers, Beijing, China, 1991. 


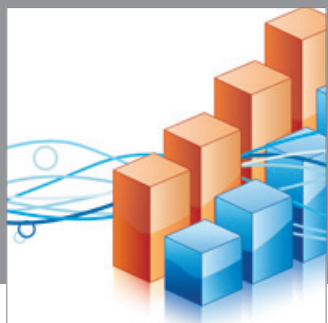

Advances in

Operations Research

mansans

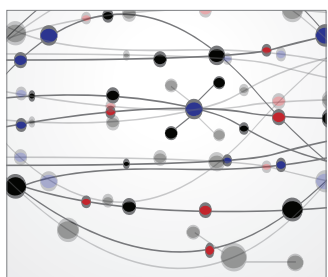

The Scientific World Journal
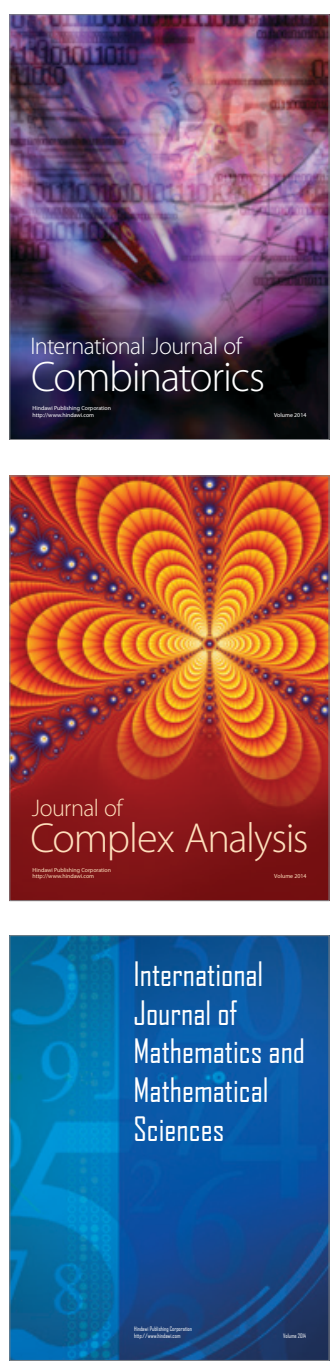
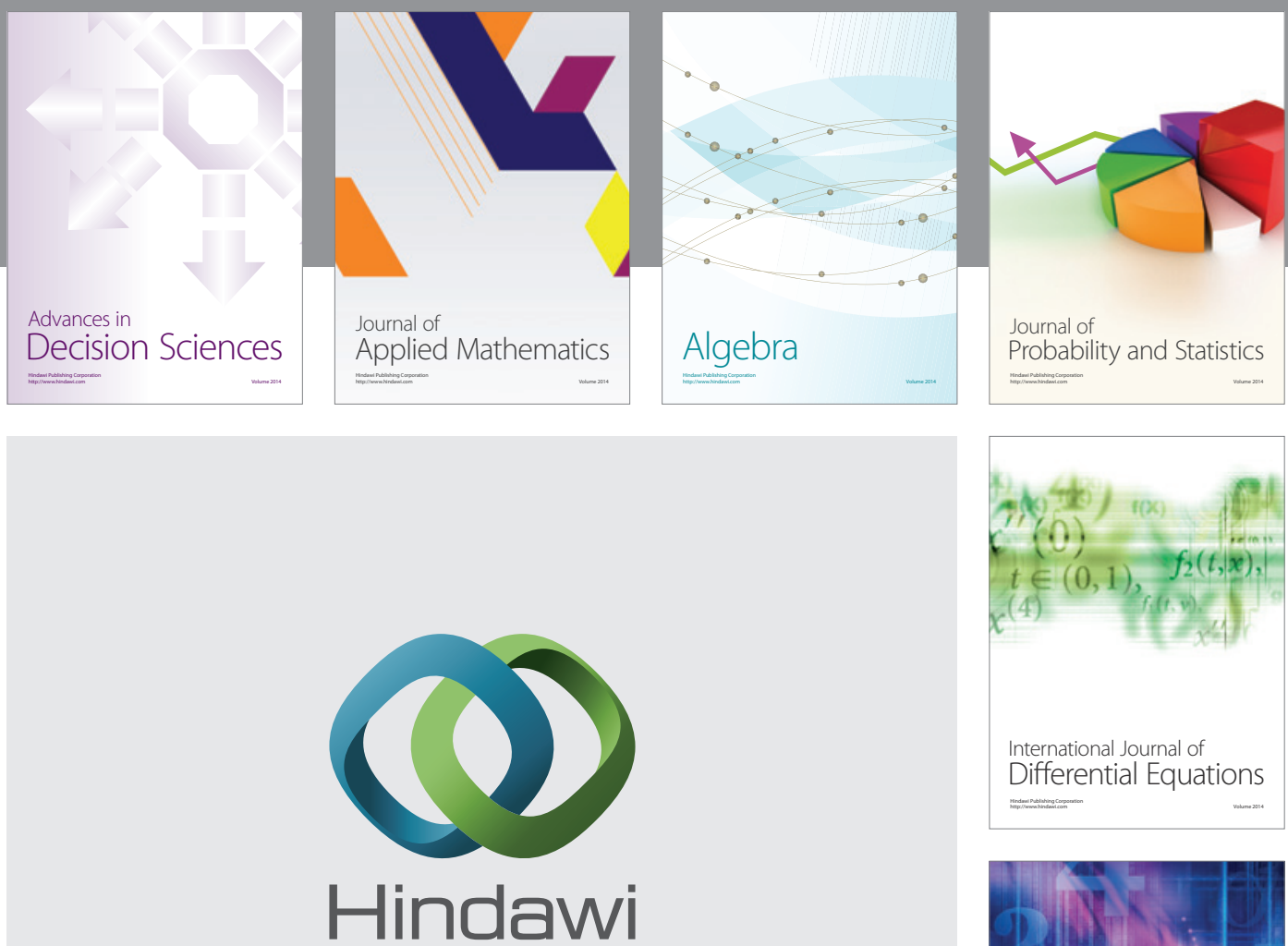

Submit your manuscripts at http://www.hindawi.com
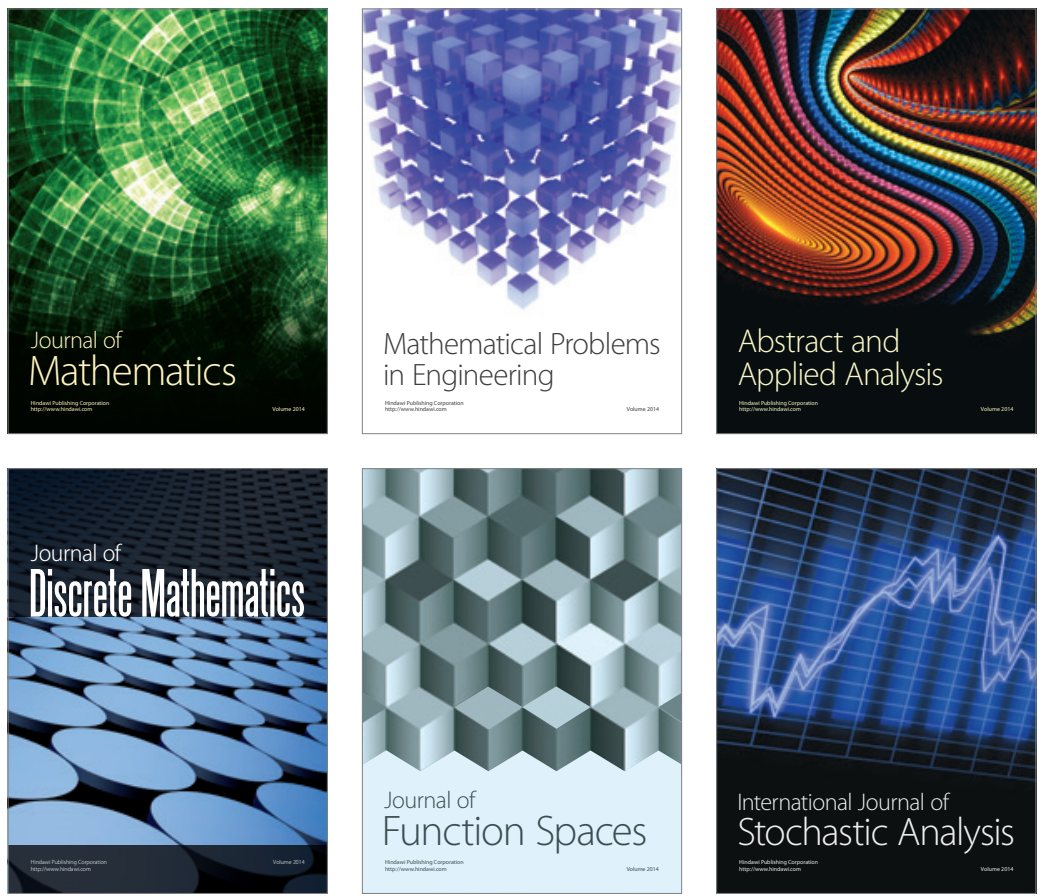

Journal of

Function Spaces

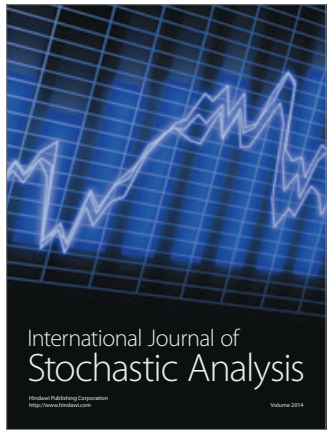

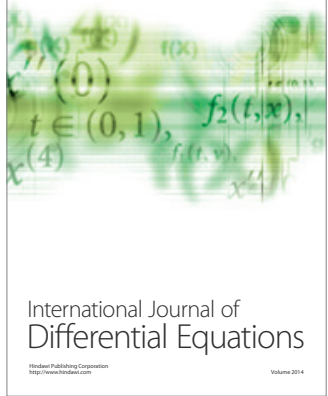
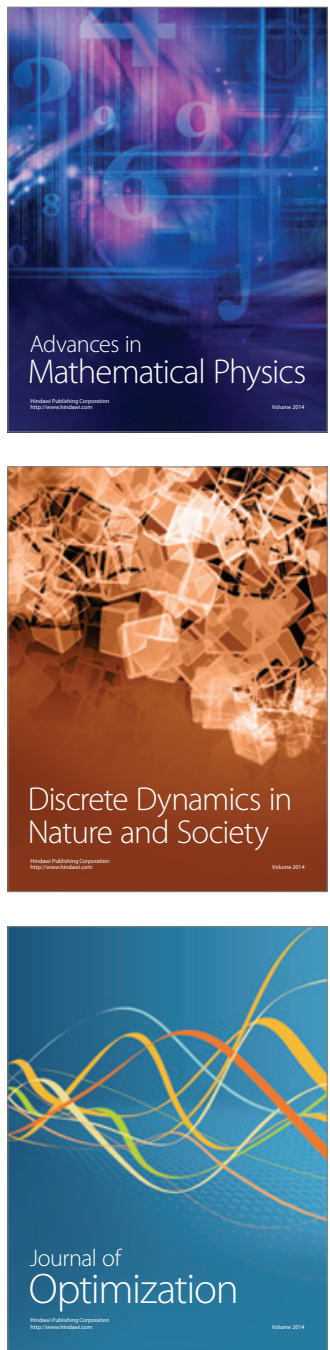